\title{
Protective properties of spliced $X$ box binding protein 1 in ozone-induced spinal cord neuronal death
}

\author{
YUN LI* ${ }^{*}$ XU ZHAO*, XIAOWEN LIN, XUEJUN ZHAO, JUNTIAN XIE, TAO SUN and ZHIJIAN FU \\ Department of Pain Management, Shandong Provincial Hospital Affiliated to Shandong University, \\ Shandong University, Jinan, Shandong 250021, P.R. China
}

Received March 13, 2017; Accepted June 7, 2018

DOI: $10.3892 / \mathrm{mmr} .2018 .9212$

\begin{abstract}
Administration of ozone $\left(\mathrm{O}_{3}\right)$ is often used in the treatment of low back pain. Administration of $\mathrm{O}_{3}$ can, however, cause neurotoxicity in spinal cord neurons via induction of endoplasmic reticulum (ER) calcium $\left(\mathrm{Ca}^{2+}\right)$ release and activation of the $\mathrm{Ca}^{2+} /$ calmodulin-dependent protein kinase II (CaMKII)/mitogen-activated protein kinase (MAPK) pathway. The aim of the current study was to confirm whether administration of $\mathrm{O}_{3}$ causes ER stress and if the consequential overexpression of adenovirus-mediated spliced $\mathrm{X}$ box binding protein 1 (XBP1s), which is the effector of ER stress and a crucial transcriptional factor gene in charge of cell survival, has a protective effect on spinal cord neurons after $\mathrm{O}_{3}$ exposure. To address this aim, the expression of GRP78, an ER chaperone and signaling regulator, and the expression of XBP1s in rat primary spinal cord neurons that underwent $\mathrm{O}_{3}$ exposure were investigated. Primary neurons exposed to $\mathrm{O}_{3}$ exhibited increased GRP78 and XBP1s expression levels. Interestingly, the effect of decreased neuron viability was blocked when cells were pretreated with Adv-XBP1s. Moreover, overexpression of XBP1s suppressed cell death caused by $\mathrm{O}_{3}$ exposure. These results suggest that overexpression of activated XBP1s protects against neuronal cell death following $\mathrm{O}_{3}$ exposure and that activation of the XBP1s pathway may offer a preventative way for prophylactic treatment of spinal cord neurons exposed to $\mathrm{O}_{3}$.
\end{abstract}

\section{Introduction}

Ozone $\left(\mathrm{O}_{3}\right)$ is a strong oxidizing agent and has been widely used in the treatment of protrusion of lumbar intervertebral disc (PLID), failed back surgery syndrome (FBSS), soft tissue

Correspondence to: Professor Zhijian Fu, Department of Pain Management, Shandong Provincial Hospital Affiliated to Shandong University, 324 Jingwu Road, Jinan, Shandong 250021, P.R. China E-mail: zhijian_fu@163.com

*Contributed equally

Key words: ozone, endoplasmic reticulum stress, glucose regulated protein78, C/EBP homologous protein, $\mathrm{X}$-box binding protein 1 lesions and arthralgia (1-3). However, Ginanneschi et al have reported ventral and dorsal root injury after intervertebral disc infiltration of $\mathrm{O}_{2} \mathrm{O}_{3}$ on L4-L5 disc herniation. Thus, physicians must distinctly comprehend the risk of potential complications (4). Therefore, whether $\mathrm{O}_{3}$ has neurotoxicity in spinal cord neurons (SCNs) has led to increasing attention to the clinical applications of $\mathrm{O}_{3}$. Previous study results suggest that exposure to $\mathrm{O}_{3}$ may cause SCNs death and that the neurotoxicity caused by $\mathrm{O}_{3}$ is related to the increasing calcium $\left(\mathrm{Ca}^{2+}\right)$ release from endoplasmic reticulum (ER), thus enhancing the activity of the $\mathrm{Ca}^{2+} /$ calmodulin-dependent protein kinase II (CaMKII)/mitogen-activated protein kinase (MAPK) pathway (5).

The ER is a multifunctional organelle that manages and participates in a wide range of cellular processes, which include protein synthesis and protein folding, handling of misfolded proteins and posttranslational modification, and delivery of proteins to their eventual destination (6). The ER forms an interconnected network of flattened, membrane-enclosed sacs in cytoplasm. It is an organelle that can provide a place to synthesize proteins and lipids. During ER stress, the intracellular folding protein and (or) unfolded protein accumulates in the ER. The cell reacts to obliterate these defective proteins, which is a process called unfolded protein response (UPR) (7). Oxidative stress damage and $\mathrm{Ca}^{+}$ depletion, which are induced by a series of toxic insults, bring about the accumulation of unfolded or misfolded proteins in the ER and lead to the dysfunction of the UPR, resulting in the onset of ER stress and cell death (8). In mammalian cells, several ER-residing transmembrane proteins have been identified as transducers of the ER stress signaling pathway, namely: Inositol-requiring ER-to-nucleus signal kinase 1 (IRE1), PKR-like ER kinase (PERK) and activating transcription factor 6 (ATF6) (9). Following activation of UPR, these transducers of ER stress are dimerized or cleaved to reduce the UPR response. In particular, dimerization and phosphorylation of IRE1 with RNase activity promotes the splicing of $\mathrm{X}$ box binding protein-1 (XBP1s) mRNA in mammalian cells. ER stress activates the RNase activity of IRE1 $\alpha$, leading to removal of 26 nucleotides from XBP1s transcripts by unorthodox RNA splicing, which transforms the inactive form of XBP1mRNA (XBP1u) into an active form (XBP1s) (10). In addition, it has been reported that processed XBP1 mRNA is rendered into the $54 \mathrm{kDa}$ processed XBP1s (11). XBP1s is 
a basic leucine zipper (bZIP)-containing transcription factor, which was initially recognized as a protein attaching to the cis-acting $\mathrm{X}$ box region in the promoter region of the human major histocompatibility complex class II genes. It is indispensable for immunoglobulin secretion and the development of plasma cells in mammals. The expression of GRP78 is induced by XBP1s through a promoter which contains the ER response element (ERSE). Accordingly, ER stress results in dimerization of PERK, resulting in the activation of PERK, thereby inducing phosphorylation of $\operatorname{IF} 2 \alpha$, the general attenuation of protein translation, and increased translation of the transcriptional factor ATF4 protein (12). The increasing translation of ATF4 can regulate the expression of $\mathrm{C} / \mathrm{EBP}$ homologous protein (CHOP), which is the downstream gene of ATF4. Both GRP78 and CHOP, which are sentinel markers for ER stress under pathologic conditions, bind to newly synthesized proteins transiently and are then translocated into the ER along with permanent unfolded or misfolded proteins (13).

It has been reported that XBP1s are effective transcription factors with diverse targets specific for the expression of ER chaperones and ER-associated degradation (ERAD) components, both of which are crucial to discharge the ER burden (14). However, ER stress may play an essential role in neuronal apoptosis, and the role of XBP1s in $\mathrm{O}_{3}$-induced ER stress remains unknown (15). The current study investigated whether exposure to low concentrations of $\mathrm{O}_{3}$ induced increased expression of GRP78 and XBP1s, and whether activation of XBP1s is neuroprotective against $\mathrm{O}_{3}$-induced ER stress.

\section{Materials and methods}

Animals and reagents. All programs involving the use of animals were conducted in adherence to the guidelines of the National Institutes of Health (NIH; Bethesda, MD, USA) and were approved by the Animal Care and Use Committee of the School of Medicine of Shandong University (Shandong, China). Neurobasal medium and B27 supplement were purchased from Gibco; Thermo Fisher Scientific, Inc. (Waltham, MA, USA). Trypsin, phenylmethylsulfonyl fluoride (PMSF) and bovine serum albumin (BSA) were obtained from Sigma-Aldrich; Merck KGaA (Darmstadt, Germany). The following primary antibodies were purchased from Cell Signaling Technology (Danvers, MA, USA): XBP1s, CHOP and GRP78. Anti- $\beta$-actin antibody was purchased from Santa Cruz Biotechnology (Santa Cruz, CA, USA).

Construction of recombinant adenovirus. The complementary DNAs encoding the full-length Rat XBP1s proteins were amplified from isolated ovary total RNA by reverse transcriptase polymerase chain reaction (RT-PCR). To clone the full-length XBP1s cDNA (GenBank acc.no. NM_005080), the following primers were used: Forward, 5'-TGAAGC TTTGCGTAGTCTGGAGCTATGG-3' and reverse, 5'-TGC TCGAGATTGGATCATTCCTTAGACA-3'. The coding reading frame of the cloned XBP1s gene was identified by sequencing and subcloned into shuttle plasmid pShuttle-CMV (Agilent, US). The constructed recombinant shuttle plasmid pShuttle-CMV-XBP1s was digested with I-CeuI and I-SceI, and the obtained expression frame of the XBP1s gene was linked to the vector carrying the pAdEasy adenovirus backbone. The recombinant adenovirus backbone was linearized by digestion with PacI and infected into HEK 293 cells for packing to obtain recombinant adenovirus Adv-XBP1s. The adenovirus was purified from the HEK 293 cell extracts by cesium chloride ( $\mathrm{CsCl}$; Nacalai Tesque, Kyoto, Japan) density ultracentrifugation according to the company manual (Takara Bio, Inc., Otsu, Japan). Viral stocks were stored in $10 \%$ glycerol at $-80^{\circ} \mathrm{C}$ and diluted with phosphate buffered saline (PBS) to the appropriate concentration prior to use. Ad-GFP, which encodes enhanced green fluorescent protein (Clontech, Palo Alto, CA), was constructed similarly by overlap recombination. The Adv-LacZ was made by the same procedure.

Primary spinal cord neuronal cultures. Primary SCNs cultures were prepared from 30 Wistar rats (1-2-days-old), as previously described (16) with minor modifications. The rats were kept by breastfeeding during a 12-h dark/light diurnal cycle. Briefly, the spinal cord from newborn rats within $48 \mathrm{~h}$ was digested for $30 \mathrm{~min}$ with trypsin $(0.125 \%$; Sigma-Aldrich; Merck KGaA). $10 \%$ fetal calf serum (FCS) was added to stop digestion and passed through a 100-eye sieve. The cell suspension was centrifuged at 1,000 rpm for $8 \mathrm{~min}$. Then, cells were washed three times with a basic culture medium with $2 \mathrm{mmol} / \mathrm{l}$ L-glutamine, penicillin (100,000 U/l), streptomycin (100 mg/l) and $10 \%$ FCS. Cells were resuspended in fresh Neurobasal medium with $2 \mathrm{mmol} / \mathrm{l} \mathrm{L-glutamine,} \mathrm{2 \%} \mathrm{(v/v)} \mathrm{B27} \mathrm{supple-}$ ment, penicillin (100,000 U/l) and streptomycin $(100 \mathrm{mg} / \mathrm{l})$. Cells were seeded on poly-L-lysine $(0.1 \mathrm{~g} / \mathrm{l})$-coated dishes at a density of $0.125 \times 10^{6}$ cells $/ \mathrm{cm}^{2}$ for the cell counting kit- 8 (CCK-8) assay or $0.33 \times 10^{6}$ cells $/ \mathrm{cm}^{2}$ for protein extraction for western blot or $0.25 \times 10^{6}$ cells $/ \mathrm{cm}^{2}$ for total RNA extraction for reverse transcription-quantitative polymerase chain reaction (RT-qPCR). For fluorescence experiments, neurons were mounted on poly-L-lysine-coated glass coverslips at a density of $0.1 \times 10^{6}$ cells $/ \mathrm{cm}^{2}$ or on poly-L-lysine-coated dishes at $0.4 \times 10^{6}$ cells $/ \mathrm{cm}^{2}$. After $24 \mathrm{~h}$, cells were treated with $0.1 \mathrm{mg} / \mathrm{ml}$ of cytosine arabinoside (Sigma-Aldrich; Merck KGaA) to suppress glia proliferation. Fresh growth medium was applied 2 times a week. Under these conditions, glia growth was $<10 \%$.

Microtubular associated protein 2 (MAP2) is specifically expressed in the neuron. We identified neurons by a positive expression of MAP2 by immunofluorescence. The cells were identified after culturing for 7 days. Briefly, cells were washed with PBS three times and fixed with $4 \%$ paraformaldehyde for $15 \mathrm{~min}$. Then, $0.2 \%$ TritonX-100 was used for cell permeabilization for $5 \mathrm{~min}$, after which cells were blocked with goat serum at room temperature for $1 \mathrm{~h}$ and incubated with anti-MAP2 monoclonal (cat. no. ab32454; 1:2,000; Abcam, Cambridge, UK) overnight at $4^{\circ} \mathrm{C}$. The next day, cells were incubated with secondary antibody (cat. no. ab150077; 1:400; Abcam) for $1 \mathrm{~h}$ in a dark room. Stained neurons were visualized using immunofluorescence microscopy.

Adenovirus transduction. Before being exposed to adenoviral vectors, the primary SCNs were maintained for two days. Culture medium was added to dilute high titer adenovirus to accomplish a multiplicity of infection (MOI) of 20-400 plaque 
forming units $(\mathrm{pfu}) / \mathrm{cell} .100 \mathrm{ml}$ of culture medium was removed and replaced with $50 \mathrm{ml}$ of culture medium with adenovirus preparation for transduction. $50 \mathrm{ml}$ of medium was added to the culture after $2 \mathrm{~h}$. Incubation with adenovirus continued for $16 \mathrm{~h}$. Then, the adenovirus medium was removed and cells were cultured in fresh culture medium. Primary SCNs were treated with adenovirus one day after plating. Cells were incubated with adenovirus at an MOI of 0-500 for $2 \mathrm{~h}$. Then, the adenovirus-containing medium was replaced with fresh medium after $2 \mathrm{~h}$.

$\mathrm{O}_{3}$ exposure. The $\mathrm{O}_{3}$ concentration was obtained through the pre-experiment. $\mathrm{CCK}-8$ assay result showed that the medical $\mathrm{O}_{3}$ of $40 \mu \mathrm{g} / \mathrm{ml}$ can reduce neuronal cell viability significantly (5). Cells were exposed to $40 \mu \mathrm{g} / \mathrm{ml} \mathrm{O}_{3}$ or tunicamycin $(2 \mu \mathrm{g} / \mathrm{ml})$ for $60 \mathrm{~min}$. SCNs exposure to $\mathrm{O}_{3}$ at specified levels were carried out in an $\mathrm{O}_{3}$ exposure chamber in vitro, which was controlled by a computer (17). The SCNs stored a thin layer of medium apically and were exposed to $\mathrm{O}_{3}$. Cells were rocked to expose one side of the apical surface at the time to $\mathrm{O}_{3}$ exposure. $\mathrm{O}_{3}$ concentration in the chamber was detected accurately by an $\mathrm{O}_{3}$ analyzer (Model MD-050-12-f-4; Perma Pure Inc., Toms River, NJ, USA) and adjusted by a computerized system.

Isolation of RNA and RT-qPCR. Total RNA was prepared by TRIzol reagent (Invitrogen; Thermo Fisher Scientific, Inc.) according to the manufacturer's instructions and was reverse transcribed to cDNA using the MML-V reverse transcriptase kit (Takara Bio, Inc.). The expression of XBP1s mRNA was examined by qPCR on a 7500-real time RT-PCR system and software (Prism 7500; Applied Biosystems Inc., Foster City, CA, USA) using a Realtime PCR Master Mix kit (Thermo Fisher Scientific, Inc., Pittsburgh, PA, USA ). qPCR was performed under the following conditions: $95^{\circ} \mathrm{C}$ for $30 \mathrm{sec}$ followed by 40 cycles of $95^{\circ} \mathrm{C}$ for $5 \mathrm{sec}$ and $60^{\circ} \mathrm{C}$ for $34 \mathrm{sec}$. GAPDH was used as an internal standard. The Prime sequences are shown as follows, GAPDH forward, 5'-CCC CCAATGTATCCGTTGTG-3' and reverse, 5'-TAGCCCAGG ATGCCCTTTAGT-3'; XBP1s forward, 5'-TGAAGCTTTGCG TAGTCTGGAGCTATGG-3' and reverse, 5'-TGCTCGAGA TTGGATCATTCCTTAGACA-3'. Samples were determined in triplicate.

Western blot analysis. Cells were collected after transfection for $24 \mathrm{~h}$. Total protein was prepared according to the manufacturer's directions. Briefly, rat SCNs were lysed in RIPA buffer with protease inhibitors (in $\mathrm{mmol} / \mathrm{l}$ : Leupeptin, 0.1 and PMSF, 0.3), and stored on ice for $30 \mathrm{~min}$ and vortexed every $5 \mathrm{~min}$. The supernatant was collected after centrifugation at $14,000 \mathrm{rpm}$ for $30 \mathrm{~min}$ at $4^{\circ} \mathrm{C}$. The protein concentration was detected by a Bio-Rad DC protein assay kit (Bio-Rad Laboratories, Inc., Hercules, CA, USA). A total of $50 \mu \mathrm{g}$ protein was loaded in each lane of standard 4-12\% SDS-polyacrylamide gels to ensure loading consistency, as previously described. Following electrophoresis and transfer of proteins to nitrocellulose membranes, the membranes were blocked in PBS containing $0.1 \%$ Tween (TBST) and 5\% non-fat milk for $1 \mathrm{~h}$. Membranes were incubated in primary anti-GRP78 (ab108613; 1:1,000),
anti-CHOP (CST; cat. no. 2895; 1:1,000) and anti-XBP1s (cat. no. ab37152; 1:1,000; all Abcam) antibody overnight at $4^{\circ} \mathrm{C}$. The membranes were incubated with horseradish peroxidase-labeled goat anti-rabbit IgG (Thermo Fisher Scientific, Inc.) as a secondary antibody $(1: 2,000)$ at room temperature for $1 \mathrm{~h}$ and then developed using ECL (GE Healthcare, Chicago, IL, USA) for detection. The bands densities were quantified by Image $\mathrm{J}$ image software v2.1.4.7 (NIH).

Evaluation of neuronal injury. Before exposure to $\mathrm{O}_{3}$ with the presence or absence of $24 \mathrm{~h} \mathrm{Adv-XBP1s}$ pretreatment, cells in 96 -well plates $\left(2 \times 10^{4}\right.$ cells/well) were washed with fresh medium and were cultured. Then, fresh medium was used to incubate the cells. Finally, the CCK-8 solution (10 $\mu \mathrm{l})$ (Dojindo Laboratories, Kumamoto, Japan) was added to each well for $2 \mathrm{~h}$ at $37^{\circ} \mathrm{C}$. Samples were measured by optical absorbance at $450 \mathrm{~nm}$ using an absorbance microplate reader (ELx800 Absorbance Microplate Reader; Bio-Tek, USA).

Statistical analysis. Data are presented as the mean \pm standard deviation(SD). The results were analyzed using one-way analysis of variance (ANOVA), followed by the Student-Newman-Keuls test for multiple comparisons with SPSS v13.0 Software (SPSS Inc., Chicago, IL, USA ). A P-value of P $\leq 0.05$ was considered statistically significant.

\section{Results}

Adenovirus mediated gene transfer into the primary SCNs. Adenovirus transduction efficiency was first optimized in the cultures. The primary SCNs were transduced with different adenovirus MOI $(5,10,50,100,150,200$, or 500) and with varying adenovirus incubation times $(2,8,18,24$ or $36 \mathrm{~h}$ ) (Fig. 1). We found the best transduction efficiency was when the primary cultures were transfected with adenovirus two days after planting and incubation for $24 \mathrm{~h}$. Under the above conditions, $83 \%$ XBP1s-positive cells could be seen in the primary SCNs three days after transduction with an MOI of 100 pfu/cell (Fig. 1A and B). Cytotoxic effects began to appear as MOI of $500 \mathrm{pfu} /$ cell.

Adenovirus mediated overexpression of XBPls in primary $S C N s$. To examine in vitro XBP1s expression levels following Ad vector infection, Western blotting and quantitative Real-time-PCR analysis were performed. We observed an apparent increase in the XBP1s expression after transduction with Adv-XBP1s in primary SCNs (Fig. 2). The control Ad vector Ad-LacZ did not increase XBP1s expression, indicating that transduction with Ad vectors alone does not induce any change in the cell systems.

$\mathrm{O}_{3}$ exposure induced the ER stress response and XBP1splicing. ER stress, which is internally linked to cell apoptosis, is one of the primary cell stress responses. Therefore, neurons are hypersensitive to ER stress challenge, and the current study investigated whether $\mathrm{O}_{3}\left(40 \mu \mathrm{g} / \mathrm{ml} \mathrm{O}_{3}\right.$ for $\left.60 \mathrm{~min}\right)$ activated the ER stress response and expression of GRP78 and CHOP, which are involved in ER stress in SCNs. As shown in Fig. 3A and B, the ER stress response of SCNs exposed to O3 was evidenced 
A
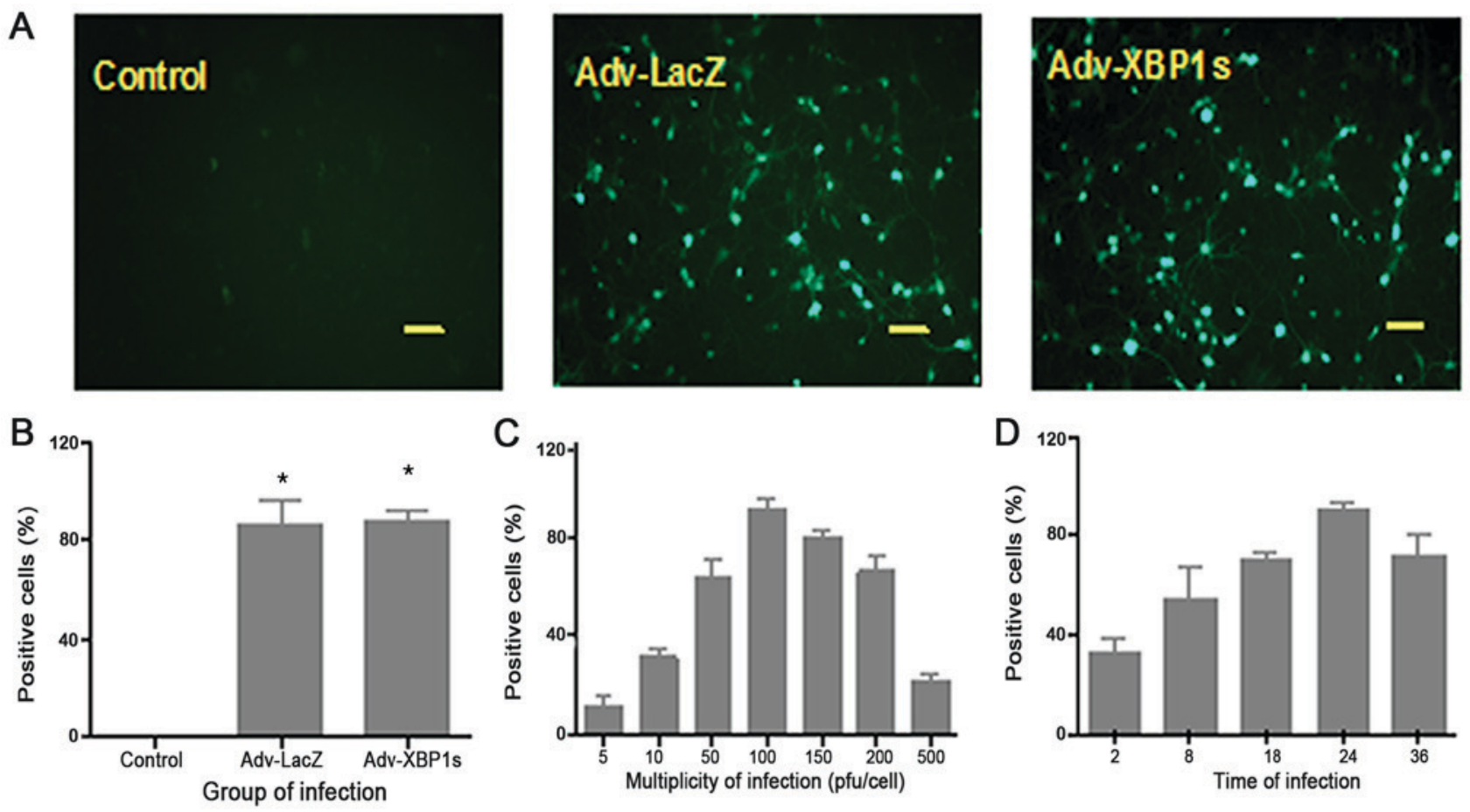

Figure 1. Determination of adenovirus transduction efficiency in primary SCNs. (A) GFP expression was observed under fluorescence microscopy (scale bar, $50 \mu \mathrm{m}$ ). (B) Transduction efficiency was estimated after infecting cells with different groups. (C) Multiplicity of infection and (D) time of infection were determined. Primary SCNs saw an increase in expression of GFP that peaked when multiplicity of infection is 100 and time is $24 \mathrm{~h}$. "P<0.05 vs. control. Control, SCNs uninfected with adenovirus; Adv-LacZ, SCNs infected with empty adenovirus; Adv-XBP1s, SCNs infected with adenovirus carrying overexpressed XBP1s. SCNs, spinal cord neurons; XBP1s, X box binding protein 1.

A

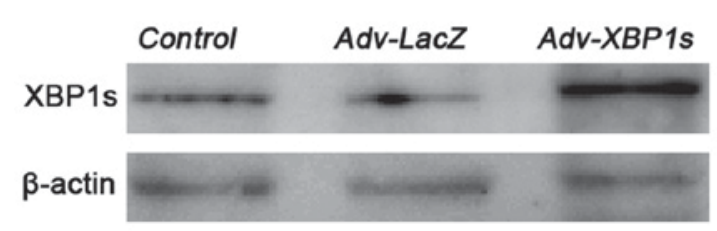

B

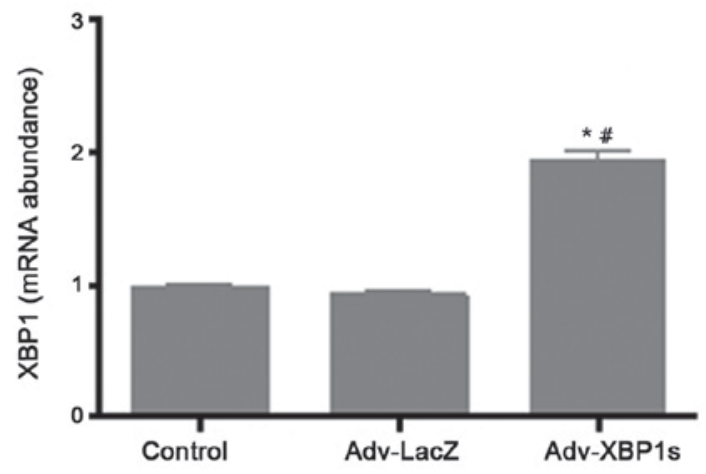

Group of infection

Figure 2. Effect of adenovirus-mediated XBP1s overexpression. (A) Western blot analysis of XBP1s protein expression in mock or stably transfected Adv-LacZ or Adv-XBP1s primary SCNs. (B) Reverse transcription-quantitative polymerase chain reaction analysis of XBP1s mRNA expression in mock or stably transfected Adv-LacZ or Adv-XBP1s primary SCNs. $\beta$-actin was used as an internal control. All experiments were performed in triplicate (n=3), "P<0.05 vs. Control; " $\mathrm{P}<0.05$ vs. Adv-LacZ. Control, SCNs uninfected with adenovirus; Adv-LacZ, SCNs infected with empty adenovirus; Adv-XBP1s, SCNs infected with adenovirus carrying overexpression of XBP1s. SCNs, spinal cord neurons; XBP1s, X box binding protein 1.

by an increase in GRP78 and CHOP protein levels. These results show that $\mathrm{O}_{3}\left(40 \mu \mathrm{g} / \mathrm{ml} \mathrm{O}_{3}\right.$ for $\left.60 \mathrm{~min}\right)$ activates the ER stress response.

Treatment of SCNs with $\mathrm{O}_{3}\left(40 \mu \mathrm{g} / \mathrm{ml} \mathrm{O}_{3}\right.$ for $\left.60 \mathrm{~min}\right)$ also increased the expression of XBP1s, as detected by a specific antibody (Fig. 3C). Analysis of XBP1 mRNA revealed $\mathrm{O}_{3}$ induced splicing of XBP1 mRNA. The splicing of XBP1 mRNA caused by $\mathrm{O}_{3}$ in SCNs was the same as that induced by the classical ER stress inducer Tunicamycin (Fig. 3D). These results indicate that $\mathrm{O}_{3}$ exposure increased XBP1s expression in SCNs.
Adenovirus-mediated XBP1s overexpression prevent ER stress and protects $\mathrm{O}_{3}$-induced cell death in rat primary $S C N$ s. During UPR, XBP1s regulate the expression of many ER stress factors that include ER chaperones and ERAD components that repair normal ER physiology. Thus, we conjecture whether XBP1s overexpression could revert the ER stress induced by $\mathrm{O}_{3}$. To detect ER stress independently of XBP1, we examined the activity of GRP78 and CHOP. Before exposure to $\mathrm{O}_{3}$, both GRP78 and CHOP expression was maintained at a lower level. However, after exposure to $\mathrm{O}_{3}$, GRP78 and CHOP expression was significantly higher (Fig. 4A and B). This suggests that 
A
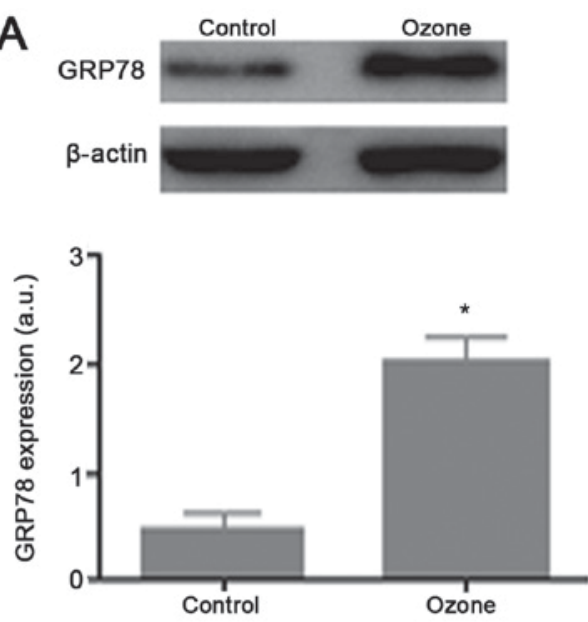

C
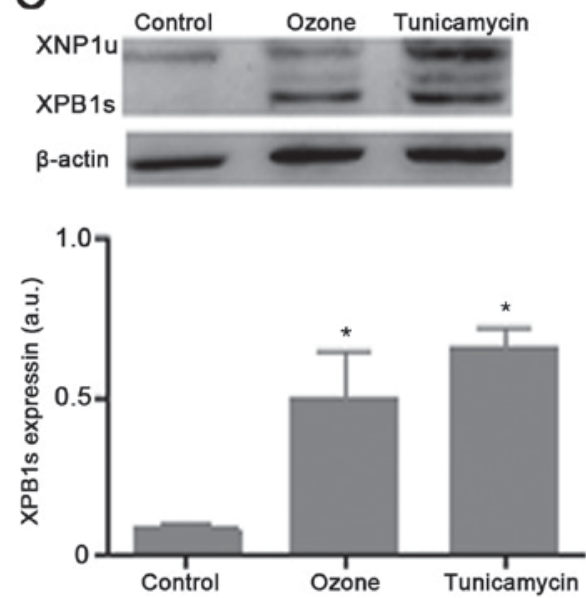

B

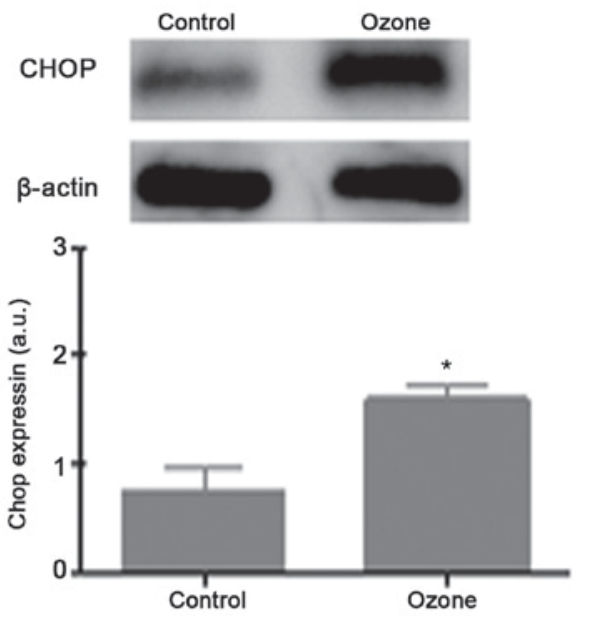

D

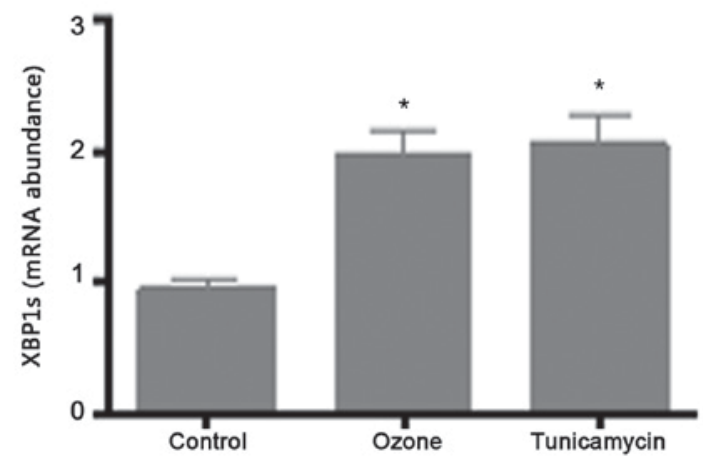

Figure 3. Expression of GRP78, CHOP and XBP1s after exposure to $\mathrm{O}_{3}$ in SCNs. (A-C) SCNs were treated for $1 \mathrm{~h}$ with $\mathrm{O}_{3}(40 \mu \mathrm{g} / \mathrm{ml})$ or tunicamycin $(2 \mu \mathrm{g} / \mathrm{ml})$. Lysates of the cells were separated by SDS-PAGE, and western blots of the membranes were detected with antibodies directed against (A) GRP78, (B) CHOP and (C) XBP1s. (D) RNA was prepared and XBP1s mRNA levels analyzed by reverse transcription-quantitative polymerase chain reaction. "P $<0.05$ vs. control; ${ }^{\#} \mathrm{P}<0.05$ vs. $\mathrm{O}_{3}$ (control, untreated control cells; $\mathrm{O}_{3}$, cells treated with $\mathrm{O}_{3}$ ). Three repeated experiments of each treatment were performed independently. Data are shown as means \pm SEM. $\mathrm{O}_{3}$, ozone; CHOP, C/EBP homologous protein; SCNs, spinal cord neurons; XBP1s, X box binding protein 1.

overexpression of XBP1s may play a neuroprotective role in ER stress.

To investigate the effect of Adv-XBP1s on $\mathrm{O}_{3}$-induced SCNs injury, SCNs were pretreated with 100MOI Adv-XBP1s for $24 \mathrm{~h}$, followed by $40 \mu \mathrm{g} / \mathrm{ml} \mathrm{O}_{3}$ treatment for $1 \mathrm{~h}$. As shown in Fig. 4, $\mathrm{O}_{3}$-induced loss of cell viability was significantly reversed by the overexpression of XBP1s. These results indicate that Adv-XBP1s have a protective effect against $\mathrm{O}_{3}$-induced toxicity in SCNs.

\section{Discussion}

Previously, $\mathrm{O}_{3}$ treatment has been widely applied in the treatment of PLID, FBSS, soft tissue lesions and arthralgia (18). The effect of $\mathrm{O}_{3}$ on the CNS, particularly biological mechanisms, has been confirmed in a previous study conducted in the authors' laboratory (5). The focus of the present study was to investigate whether there is a novel and effective method to prevent neurotoxicity induced by $\mathrm{O}_{3}$. In the present study, $\mathrm{SCNs}$ were exposed to $\mathrm{O}_{3}$ to induce GRP78 and XBP1 activation, resulting in increased cell death. Interestingly, pretreatment of cells with Adv-XBP1s inhibited these effects. The aim of the current study was to provide insight into the protective role of XBP1s overexpression. To address this aim, the role of XBP1s, a critical UPR effector, was investigated using an in vitro rat primary SCNs model of $\mathrm{O}_{3}$ injury.

This study focuses on the protective role of XBP1s in $\mathrm{O}_{3}$-induced spinal cord neuronal death. First, ER stress was activated during $\mathrm{O}_{3}$-induced $\mathrm{SCNs}$ death. As a result, an increase in GRP78 and CHOP levels as well as extensive splicing of XBP1s appeared upon $\mathrm{O}_{3}$ treatment (Fig. 3A-C). Second, SCNs with XBP1s overexpression prevented cell death, and partially reduced the protein levels of GRP78 and CHOP (Fig. 4A and B). Strong activation of GRP78 was also observed in previous studies during tunicamycin (an inhibitor of N-linked glycosylation) and thapsigargin (an inhibitor of the ER $\mathrm{Ca}^{2+}$-ATPase) treatment. In our study, we observed a similar result where the transcription and protein expression levels of XBP1s in SCNs were significantly increased after tunicamycin treatment (Fig. 3C and D). As a sentinel marker for ER stress under pathologic conditions, GRP78 binds transiently to newly synthesized proteins translocated into the ER and more permanently to unfolded or misfolded proteins (19). GRP78 can trigger the UPR via dissociation from its interaction partners 

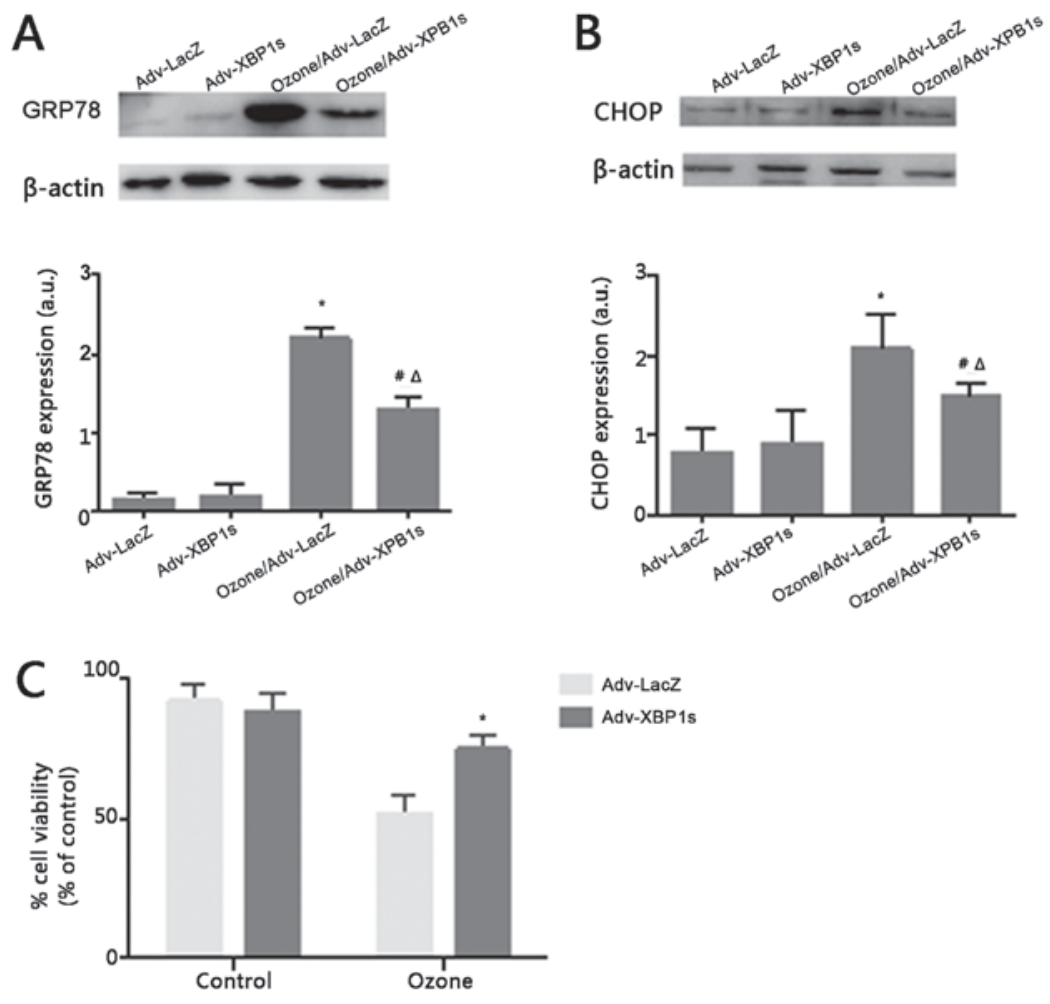

Figure 4. Protective effect of XBP1s overexpression in primary SCNs against $\mathrm{O}_{3}$. Western blot analysis indicated (A) GRP78 and (B) CHOP expression levels were significantly increased after primary SCNs exposed to $\mathrm{O}_{3}(40 \mu \mathrm{g} / \mathrm{ml})$. But the role may have been reverted by overexpression of XBP1s. "P $<0.05 \mathrm{vs}$. Adv-LacZ; ${ }^{\#} \mathrm{P}<0.05$ vs. Adv-XBP1s, ${ }^{\Delta} \mathrm{P}<0.05$ vs. $\mathrm{O}_{3} / \mathrm{Adv}-\mathrm{XBP} 1 \mathrm{~s}$. (C) Cell viability was determined using CCK-8 after $\mathrm{O}_{3}$ exposure. Mean $\pm \mathrm{SD}$ is shown (n=9). $\mathrm{XBP} 1 \mathrm{~s}$ transfected cells (Adv-XBP1s) exhibited increased viability ${ }^{*} \mathrm{P}<0.05$ vs. cells treated with $\mathrm{O}_{3} / \mathrm{Adv}$-LacZ. All data were obtained through three independently repeated experiments. CCK-8, cell counting kit-8; $\mathrm{O}_{3}$, ozone; CHOP, C/EBP homologous protein; $\mathrm{SCNs}$, spinal cord neurons; XBP1s, X box binding protein 1 .

PERK, ATF6 and IRE1a, which can subsequently lead to activation of ER stress responses involving an induced expression of ER chaperones to increase the folding capacity of the ER. When ER stress occurs, sensor proteins from the ER membrane are dimerized or cleaved to weaken this condition. IRE1, which is a sensor protein with RNase activity, is dimerized and phosphorylated to expedite splicing of mRNA for XBP1 in mammalian cells $(14,20)$. XBP1s protein from spliced mRNA induces GRP78 expression via a promoter containing the ERSE.

In addition, CHOP is a transcription factor belonging to the (c/EBP) family that induces cell cycle arrest and apoptosis in ER stress and DNA damage response (21). Basal expression of CHOP is very low under non-stressed conditions; however, its expression is induced by a number of adverse physiological conditions. The expression of CHOP is both cell- and stimulus-dependent, and thus has an influence on the final result of ER stress. The expression of CHOP is strongly induced via IRE1 and PERK signaling (22). Our research showed that $\mathrm{SCNs}$ exposed to $\mathrm{O}_{3}$ had clearly raised the expression levels of CHOP, which is an ER-related chaperone (Fig. 3A and B). This suggested that $\mathrm{O}_{3}(40 \mu \mathrm{g} / \mathrm{ml})$ might induce SCNs death through ER stress and DNA damage.

In the UPR, the expressions of ER chaperones and ERAD-related genes appear to be largely regulated by the IRE1 $\alpha$-XBP1s pathway $(20,23)$. The activated form of XBP1s upregulates many ER chaperones and genes involved in ERAD, various UPR 'stress genes', and enzymes involved in membrane biogenesis (24). In addition, the overexpression of XBP1s plays a protective role in a neuroblastoma cell line against cell death, which is induced by proteasome inhibition (25). XBP1s overexpression protected against oxygen and glucose deprivation/reoxygenation injury in rat primary hippocampal neurons (26). This is meet with our research. Besides, a recent study showed that overexpression of XBP1s can promote proliferation in bone marrow-derived macrophages (27), which is in accordance with our results. Our research work found that overexpression of XBP1s protected SCNs from $\mathrm{O}_{3}$-induced cell death, which may play a protective role through the IRE1 $\alpha$-XBP1 pathway. Whether the protective role of overexpression of XBP1s is involved in some ER-related genes or enzymes will be investigated in future research.

Taken together, it was hypothesized that the activation of the XBP1s pathway and its downstream ER chaperones could prevent cytotoxicity and play a protective effect. Therefore, the impact on the activation of the IRE1 $\alpha$-XBP1s pathway in opposition to cell death induced by these damages in addition to representative ER stressors were investigated. As expected, pretreatment with adenovirus-mediated overexpression of XBP1s strongly reduced SCNs death induced by $\mathrm{O}_{3}$.

These results suggest that XBP1s activation could potentially be applied to prevent ER stress-related neurotoxicity and this cytoprotective effect was confirmed by XBP1s overexpression. Furthermore, XBP1s and other components of the ER stress response pathway could serve as potential drug targets in preventative strategies for neurotoxicity associated with ER stress. While the current study provides in vitro data regarding the protective role of overexpression of XBP1 in SCNs, in vivo therapeutic implications are still required. 


\section{Acknowledgements}

The authors would like to thank the Central Laboratory affiliated Shandong Provincial Hospital (Jinan, China).

\section{Funding}

The present study was funded by grants from the National Natural Science Foundation of China (grant no. 81271346) and the Natural Science Foundation of Shandong Province, China (grant no. ZR2010HM097).

\section{Availability of data and materials}

The datasets used and/or analyzed during the current study are available from the corresponding author on reasonable request.

\section{Authors' contributions}

$\mathrm{ZF}, \mathrm{XL}$ and $\mathrm{YL}$ conceived and designed the experiments. YL and XZ performed the experiments. XJZ, JX and TS conducted data analysis. YL and XZ produced the manuscript.

\section{Ethics approval and consent to participate}

All animal experiments were approved by Animal Care and Use Committee of Shandong Provincial Hospital Affiliated to Shandong University.

\section{Patient consent for publication}

Not applicable.

\section{Competing interests}

All authors declare that they have no competing interests.

\section{References}

1. Elvis AM and Ekta JS: Ozone therapy: A clinical review. J Nat Sci Biol Med 2: 66-70, 2011

2. Paoloni M, Di Sante L, Cacchio A, Apuzzo D, Marotta S, Razzano M, Franzini $M$ and Santilli V: Intramuscular oxygen-ozone therapy in the treatment of acute back pain with lumbar disc herniation: A multicenter, randomized, double-blind, clinical trial of active and simulated lumbar paravertebral injection. Spine (Phila Pa 1976) 34: 1337-1344, 2009.

3. Zhang Y, Ma Y, Jiang J, Ding T and Wang J: Treatment of the lumbar disc herniation with intradiscal and intraforaminal injection of oxygen-ozone. J Back Musculoskelet Rehabil 26: 317-322, 2013.

4. Ginanneschi F, Cervelli C, Milani P and Rossi A: Ventral and dorsal root injury after oxygen-ozone therapy for lumbar disk herniation. Surg Neurol 66: 619-621, 2006.

5. Li Y, Lin X, Zhao X, Xie J, JunNan W, Sun T and Fu Z: Ozone (O3) elicits neurotoxicity in spinal cord neurons (SCNs) by inducing $\mathrm{ER} \mathrm{Ca}(2+)$ release and activating the $\mathrm{CaMKII} / \mathrm{MAPK}$ signaling pathway. Toxicol Appl Pharmacol 280: 493-501, 2014.

6. Hetz C: The unfolded protein response: controlling cell fate decisions under ER stress and beyond. Nat Rev Mol Cell Biol 13: 89-102, 2012.
7. Verkhratsky A and Petersen $\mathrm{OH}$ : The endoplasmic reticulum as an integrating signalling organelle: From neuronal signalling to neuronal death. Eur J Pharmacol 447: 141-154, 2002.

8. Tang CH, Chiu YC, Huang CF, Chen YW and Chen PC: Arsenic induces cell apoptosis in cultured osteoblasts through endoplasmic reticulum stress. Toxicol Appl Pharmacol 241: 173-181, 2009.

9. Zhang K and Kaufman RJ: Signaling the unfolded protein response from the endoplasmic reticulum. J Biol Chem 279: 25935-25938, 2004.

10. Lin JH, Li H, Yasumura D, Cohen HR, Zhang C, Panning B, Shokat KM, Lavail MM and Walter P: IRE1 signaling affects cell fate during the unfolded protein response. Science 318: 944-949, 2007.

11. Paschen W, Aufenberg C, Hotop S and Mengesdorf T: Transient cerebral ischemia activates processing of $\mathrm{xbpl}$ messenger RNA indicative of endoplasmic reticulum stress. J Cereb Blood Flow Metab 23: 449-461, 2003

12. Lu PD, Harding HP and Ron D: Translation reinitiation at alternative open reading frames regulates gene expression in an integrated stress response. J Cell Biol 167: 27-33, 2004.

13. Yoshida $H$, Yanagi $H$, Yura $T$ and Mori K: Identification of the cis-acting endoplasmic reticulum stress response element responsible for transcriptional induction of mammalian glucose-regulate proteins. Involvement of basic leucine zipper transcription factors. J Biol Chem 273: 33741-33749, 1998.

14. Yoshida H: Unconventional splicing of XBP-1 mRNA in the unfolded protein response. Antioxid Redox Signal 9: 2323-33, 2007.

15. Chen J, Qin J, Liu X, Han Y, Yang Z, Chang X and Ji X: Nitric oxide-mediated neuronal apoptosis in rats with recurrent febrile seizures through endoplasmic reticulum stress pathway. Neurosci Lett 443: 134-139, 2008.

16. Marsala M, Kakinohana O, Hefferan MP, Cizkova D, Kinjoh K and Marsala S: Synaptogenesis and amino acid release from long term embryonic rat spinal cord neuronal culture using tissue culture inserts. J Neurosci Methods 141: 21-27, 2005.

17. Ahmad A, Ahmad S, Chang LY, Schaack J and White CW: Endothelial Akt activation by hyperoxia: Role in cell survival. Free Radic Biol Med 40: 1108-1118, 2006.

18. Muto M, Ambrosanio G, Guarnieri G, Capobianco E, Piccolo G, Annunziata G and Rotondo A: Low back pain and sciatica: Treatment with intradiscal-intraforaminal $\mathrm{O}$ (2)-O (3) injection. Our experience. Radiol Med 113: 695-706, 2008 (In English, Italian).

19. Yoshida H, Matsui T, Yamamoto A, Okada T and Mori K: XBP1 mRNA is induced by ATF6 and spliced by IRE1 in response to ER stress to produce a highly active transcription factor. Cell 107: 881-891, 2001.

20. Lee AE, Iwakoshi NN and Glimcher LH: XBP-1 regulates a subset of endoplasmic reticulum resident chaperone genes in the unfolded protein response. Mol Cell Biol 23: 7448-7459, 2003.

21. Oyadomari S and Mori M: Roles of CHOP/GADD153 in endoplasmic reticulum stress. Cell Death Differ 11: 381-389, 2004.

22. Mihailidou C, Papazian I, Papavassiliou AG and Kiaris H: CHOP-dependent regulation of p21/waf1 during ER stress. Cell Physiol Biochem 25: 761-766, 2010.

23. Yamauchi T, Sakurai M, Abe K, Matsumiya G and Sawa Y: Impact of the endoplasmic reticulum stress response in spinal cord after transient ischemia. Brain Res 1169: 24-33, 2007.

24. Ron D and Walter P: Signal integration in the endoplasmic reticulum unfolded protein response. Nat Rev Mol Cell Biol 8: 519-529, 2007.

25. Sado M, Yamasaki Y, Iwanaga T, Onaka Y, Ibuki T, Nishihara S, Mizuguchi H, Momota H, Kishibuchi R, Hashimoto T, et al: Protective effect against Parkinson's disease-related insults through the activation of XBP1. Brain Res 1257: 16-24, 2009.

26. Ibuki T, Yamasaki Y, Mizuguchi H and Sokabe M: Protective effects of XBP1 against oxygen and glucose deprivation/reoxygenation injury in rat primary hippocampal neurons. Neurosci Lett 518: 45-48, 2012.

27. Tian PG, Jiang ZX, Li JH, Zhou Z and Zhang QH, Spliced XBP1 promotes macrophage survival and autophagy by interacting with Beclin-1. Biochem Biophys Res Commun 463: 518-523, 2015. 